J. Korean Math. Soc. 47 (2010), No. 4, pp. 705-718

DOI 10.4134/JKMS.2010.47.4.705

\title{
LIE BIALGEBRAS ARISING FROM POISSON BIALGEBRAS
}

\author{
Sei-Qwon Oh And Eun-Hee Cho
}

\begin{abstract}
It gives a method to obtain a natural Lie bialgebra from a Poisson bialgebra by an algebraic point of view. Let $\mathfrak{g}$ be a coboundary Lie bialgebra associated to a Poission Lie group $G$. As an application, we obtain a Lie bialgebra from a sub-Poisson bialgebra of the restricted dual of the universal enveloping algebra $U(\mathfrak{g})$.
\end{abstract}

\section{Introduction}

Assume throughout that $G$ denotes a connected and simply connected Lie group with Lie algebra $\mathfrak{g}, \mathcal{O}(G)$ the coordinate $\operatorname{ring}$ of $G$ and $U(\mathfrak{g})$ the universal enveloping algebra of $\mathfrak{g}$.

If $G$ is a Poisson Lie group, then $\mathcal{O}(G)$ is a Poisson Hopf algebra and $\mathfrak{g}$ becomes a Lie bialgebra. Conversely, if $\mathfrak{g}$ has a Lie bialgebra structure, then $G$ becomes a Poisson Lie group by [2, Chapter 1]. On the other hand, if $U(\mathfrak{g})$ has a co-Poisson Hopf structure with co-Poisson bracket $\delta$, then $\left(\mathfrak{g},\left.\delta\right|_{\mathfrak{g}}\right)$ becomes a Lie bialgebra. Conversely if $(\mathfrak{g}, \delta)$ is a Lie bialgebra, then the cobracket $\delta$ extends uniquely to a Poisson co-bracket on $U(\mathfrak{g})$, which makes $U(\mathfrak{g})$ into a co-Poisson Hopf algebra (see [2, Proposition 6.2.3]). Moreover, the coordinate ring $\mathcal{O}(G)$ is isomorphic as a Hopf algebra to the restricted dual $U(\mathfrak{g})^{\circ}$ of $U(\mathfrak{g})$ and it is sometimes more convenient to work on $U(\mathfrak{g})^{\circ}$ than to do on $\mathcal{O}(G)$. For instance, Hodges and his colleagues worked on restricted duals to obtain mathematical properties of a quantum group in [3] and [4]. Hence it makes sense mathematically to study a relationship between Lie bialgebras and restricted duals of their enveloping algebras.

Let $(A, \iota, m,\{\cdot, \cdot\}, \epsilon, \Delta)$ be a Poisson bialgebra and $\mathfrak{m}=\operatorname{ker} \epsilon$. In 1.5 , we prove by an algebraic point of view that the pair $\left(\left(\mathfrak{m} / \mathfrak{m}^{2}\right)^{*}, \mathfrak{m} / \mathfrak{m}^{2}\right)$ is a natural Lie bialgebra obtained from $(A, \iota, m,\{\cdot, \cdot\}, \epsilon, \Delta)$.

Let $\mathfrak{g}$ be a coboundary Lie bialgebra. The restricted dual $A$ of $U(\mathfrak{g})$ is the vector space spanned by all coordinate functions $c_{f, v}^{M}$, where $M$ is a finite

Received January 31, 2008.

2000 Mathematics Subject Classification. 17B62, 17B63, 16W30.

Key words and phrases. Lie bialgebra, Poisson bialgebra.

This work was supported by the Korea Research Foundation Grant funded by the Korean Government, KRF-2007-314-C00007. 
dimensional left $U(\mathfrak{g})$-module and $f \in M^{*}, v \in M$. Here we give an explicit Poisson bracket on $A$ that is the Sklyanin Poisson bracket. Let $B$ be a subPoisson bialgebra of the restricted dual $A$. Then, as an application of 1.5 , we obtain a Lie bialgebra $\left(\left(\mathfrak{m}_{B} / \mathfrak{m}_{B}^{2}\right)^{*}, \mathfrak{m}_{B} / \mathfrak{m}_{B}^{2}\right)$ arising from $B$, where $\mathfrak{m}_{B}$ is the kernel of the counit in $B$.

Assume throughout that $\mathbf{k}$ denotes a field of characteristic zero, all vector spaces considered here are over $\mathbf{k}$ and if $A$ is a bialgebra with comultiplication $\Delta$, then we use Sweedler's notation

$$
\Delta(a)=\sum_{(a)} a^{\prime} \otimes a^{\prime \prime}, \quad a \in A .
$$

Recall that a Poisson algebra $A$ is a $\mathbf{k}$-algebra with $\mathbf{k}$-bilinear map $\{\cdot, \cdot\}$, called a Poisson bracket, such that

(a) $(A,\{\cdot, \cdot\})$ is a Lie algebra over $\mathbf{k}$.

(b) $\{a b, c\}=a\{b, c\}+\{a, c\} b$ for all $a, b, c \in A$. (Leibniz rule)

\section{Lie bialgebra arising from Poisson bialgebra}

Definition 1.1. A Poisson algebra $A$ with Poisson bracket $\{\cdot, \cdot\}$ is said to be a Poisson bialgebra if $A$ is also a bialgebra $(A, \iota, m, \epsilon, \Delta)$ over $\mathbf{k}$ such that

$$
\Delta(\{a, b\})=\{\Delta(a), \Delta(b)\}_{A \otimes A}
$$

for all $a, b \in A$, where the Poisson bracket $\{\cdot, \cdot\}_{A \otimes A}$ on $A \otimes A$ is defined by

$$
\{a \otimes b, c \otimes d\}_{A \otimes A}=\{a, c\} \otimes b d+a c \otimes\{b, d\}
$$

for all $a, b, c, d \in A$.

A Poisson bialgebra $A$ is often denoted by $A=(A, \iota, m,\{\cdot, \cdot\}, \epsilon, \Delta)$. If a Poisson bialgebra $A$ is a Hopf algebra, then $A$ is called a Poisson Hopf algebra (see [2, 6.2.1] and [1, III.5.3]). Note, by (1), that

$$
\Delta(\{a, b\})=\sum a^{\prime} b^{\prime} \otimes\left\{a^{\prime \prime}, b^{\prime \prime}\right\}+\left\{a^{\prime}, b^{\prime}\right\} \otimes a^{\prime \prime} b^{\prime \prime}
$$

for all elements $a, b$ of a Poisson bialgebra $A$.

Lemma 1.2. If $(A, \iota, m,\{\cdot, \cdot\}, \epsilon, \Delta)$ is a Poisson bialgebra, then $\epsilon(\{a, b\})=0$ for all $a, b \in A$.

Proof. By (2), we have that

$$
\begin{aligned}
\{a, b\} & =m \circ\left(\epsilon \otimes \operatorname{id}_{A}\right) \circ \Delta(\{a, b\}) \\
& =m \circ\left(\epsilon \otimes \operatorname{id}_{A}\right)\left(\sum\left\{a^{\prime}, b^{\prime}\right\} \otimes a^{\prime \prime} b^{\prime \prime}+a^{\prime} b^{\prime} \otimes\left\{a^{\prime \prime}, b^{\prime \prime}\right\}\right) \\
& =\sum \epsilon\left(\left\{a^{\prime}, b^{\prime}\right\}\right) a^{\prime \prime} b^{\prime \prime}+\sum \epsilon\left(a^{\prime} b^{\prime}\right)\left\{a^{\prime \prime}, b^{\prime \prime}\right\} \\
& =\sum \epsilon\left(\left\{a^{\prime}, b^{\prime}\right\}\right) a^{\prime \prime} b^{\prime \prime}+\{a, b\}
\end{aligned}
$$

for $a, b \in A$ and thus we have $\sum \epsilon\left(\left\{a^{\prime}, b^{\prime}\right\}\right) a^{\prime \prime} b^{\prime \prime}=0$. Hence

$$
0=\epsilon\left(\sum \epsilon\left(\left\{a^{\prime}, b^{\prime}\right\}\right) a^{\prime \prime} b^{\prime \prime}\right)=\sum \epsilon\left(\left\{a^{\prime}, b^{\prime}\right\}\right) \epsilon\left(a^{\prime \prime}\right) \epsilon\left(b^{\prime \prime}\right)=\epsilon(\{a, b\}),
$$


as claimed.

Corollary 1.3. In a Poisson bialgebra $(A, \iota, m,\{\cdot, \cdot\}, \epsilon, \Delta)$, set $\operatorname{ker} \epsilon=\mathfrak{m}$. Then $\mathfrak{m} / \mathfrak{m}^{2}$ is a Lie algebra with Lie bracket

$$
\left[a+\mathfrak{m}^{2}, b+\mathfrak{m}^{2}\right]=\{a, b\}+\mathfrak{m}^{2}, \quad a, b \in \mathfrak{m} .
$$

Proof. The Lie bracket (3) is well-defined by Lemma 1.2. Clearly $\left(\mathfrak{m} / \mathfrak{m}^{2},[\cdot, \cdot]\right)$ is a Lie algebra.

1.4. Let $(A, \iota, m, \epsilon, \Delta)$ be a bialgebra and set

$$
A^{\circ}=\left\{f \in A^{*} \mid f(I)=0 \text { for some ideal } I \text { of } A \text { such that } \operatorname{dim}(A / I)<\infty\right\} .
$$

Then $A^{\circ}$, called the restricted dual of $A$, becomes a bialgebra with bialgebra structure: For $f, g \in A^{\circ}$ and $a, b \in A$,

$$
(f g)(a)=\sum f\left(a^{\prime}\right) g\left(a^{\prime \prime}\right), \quad \Delta(f)(a \otimes b)=f(a b) .
$$

Denote

$$
P_{\epsilon}\left(A^{\circ}\right)=\left\{f \in A^{\circ} \mid f(a b)=\epsilon(a) f(b)+f(a) \epsilon(b), \quad \forall a, b \in A\right\} .
$$

That is, $P_{\epsilon}\left(A^{\circ}\right)=\left\{f \in A^{\circ} \mid \Delta(f)=\epsilon \otimes f+f \otimes \epsilon\right\}$. It is well-known that $P_{\epsilon}\left(A^{\circ}\right)$ is a Lie algebra with Lie bracket

$$
[f, g]=f g-g f
$$

for all $f, g \in P_{\epsilon}\left(A^{\circ}\right)$.

Denote $\mathfrak{m}=\operatorname{ker} \epsilon$ and let $i: \mathfrak{m} \longrightarrow A$ be the canonical injection. Then $i^{*}$ is a surjection of $A^{*}$ onto $\mathfrak{m}^{*}$. Let $f \in \operatorname{ker} i^{*}$. Then $f(a-\epsilon(a) 1)=0$ for all $a \in A$ since $f(\mathfrak{m})=0$ and $a-\epsilon(a) 1 \in \mathfrak{m}$. Thus $f=f(1) \epsilon$ for $f \in \operatorname{ker} i^{*}$. It follows that ker $i^{*}=\mathbf{k} \epsilon$. Given $f, g \in \mathfrak{m}^{*}$, choose representatives $f^{\prime}=i^{*-1}(f), g^{\prime}=i^{*-1}(g)$. If $f_{1}^{\prime}=i^{*-1}(f), g_{1}^{\prime}=i^{*-1}(g)$, then $f_{1}^{\prime}=f^{\prime}+\alpha \epsilon, g_{1}^{\prime}=g^{\prime}+\beta \epsilon$ for some $\alpha, \beta \in \mathbf{k}$. Thus

$$
\begin{aligned}
f_{1}^{\prime} g_{1}^{\prime}-g_{1}^{\prime} f_{1}^{\prime} & =\left(f^{\prime}+\alpha \epsilon\right)\left(g^{\prime}+\beta \epsilon\right)-\left(g^{\prime}+\beta \epsilon\right)\left(f^{\prime}+\alpha \epsilon\right) \\
& =\left(f^{\prime} g^{\prime}+\beta f^{\prime}+\alpha g^{\prime}+\alpha \beta \epsilon\right)-\left(g^{\prime} f^{\prime}+\beta f^{\prime}+\alpha g^{\prime}+\alpha \beta \epsilon\right) \\
& =f^{\prime} g^{\prime}-g^{\prime} f^{\prime}
\end{aligned}
$$

since $\epsilon$ is the multiplicative identity in $A^{*}$. Hence $[f, g]=i^{*}\left(f^{\prime} g^{\prime}-g^{\prime} f^{\prime}\right)$ is independent of representatives and defines a Lie bracket on $\mathfrak{m}^{*}$. Identifying $\left\{f \in \mathfrak{m}^{*} \mid f\left(\mathfrak{m}^{2}\right)=0\right\}$ with $\left(\mathfrak{m} / \mathfrak{m}^{2}\right)^{*},\left(\mathfrak{m} / \mathfrak{m}^{2}\right)^{*}$ is a Lie subalgebra of $\mathfrak{m}^{*}$ by $[8$, $2.1 .2]$.

Lemma. The linear map

$$
\left.i^{*}\right|_{P_{\epsilon}\left(A^{\circ}\right)}: P_{\epsilon}\left(A^{\circ}\right) \longrightarrow\left(\mathfrak{m} / \mathfrak{m}^{2}\right)^{*},\left.\quad f \mapsto i^{*}\right|_{P_{\epsilon}\left(A^{\circ}\right)}(f)=\left.f\right|_{\mathfrak{m}}
$$

is a Lie isomorphism. 
Proof. Note that $A=\mathbf{k} 1_{A} \oplus \mathfrak{m}$ and if $f \in P_{\epsilon}\left(A^{\circ}\right)$, then $f\left(\mathfrak{m}^{2}\right)=0$. Hence $\left.i^{*}\right|_{P_{\epsilon}\left(A^{\circ}\right)}$ is well-defined. If $f \in \operatorname{ker}\left(\left.i^{*}\right|_{P_{\epsilon}\left(A^{\circ}\right)}\right)$, then

$$
f\left(\alpha 1_{A}+a\right)=\alpha f\left(1_{A}\right)+f(a)=0
$$

for all $\alpha \in \mathbf{k}$ and $a \in \mathfrak{m}$. It follows that $\left.i^{*}\right|_{P_{\epsilon}\left(A^{\circ}\right)}$ is injective. If $f \in \mathfrak{m}^{*}$ such that $f\left(\mathfrak{m}^{2}\right)=0$, then $f$ is extended to $A$, denoted by $f^{\prime}$, by setting

$$
f^{\prime}\left(\mathbf{k} 1_{A}\right)=0,\left.\quad f^{\prime}\right|_{\mathfrak{m}}=f .
$$

Then, for any $\alpha, \beta \in \mathbf{k}$ and $a, b \in \mathfrak{m}$,

$$
\begin{aligned}
f^{\prime}\left(\left(\alpha 1_{A}+a\right)\left(\beta 1_{A}+b\right)\right) & =f(\alpha b)+f(\beta a) \\
& =\epsilon\left(\alpha 1_{A}+a\right) f^{\prime}\left(\beta 1_{A}+b\right)+f^{\prime}\left(\alpha 1_{A}+a\right) \epsilon\left(\beta 1_{A}+b\right) .
\end{aligned}
$$

Hence $f^{\prime} \in P_{\epsilon}\left(A^{\circ}\right)$ and thus $\left.i^{*}\right|_{P_{\epsilon}\left(A^{\circ}\right)}$ is surjective. Now $\left.i^{*}\right|_{P_{\epsilon}\left(A^{\circ}\right)}$ is a Lie isomorphism by the definition of Lie brackets.

1.5. Let us recall the definition for Lie bialgebra in $[2,1.3]$ and $[9,2.1 .1]$. A Lie bialgebra is a pair $(\mathfrak{g}, \psi)$, where $\mathfrak{g}$ is a Lie algebra and $\psi: \mathfrak{g} \longrightarrow \mathfrak{g} \wedge \mathfrak{g}$, called cobracket, satisfying the following conditions:

(a) The dual map $\psi^{*}: \mathfrak{g}^{*} \wedge \mathfrak{g}^{*} \longrightarrow \mathfrak{g}^{*}$ makes $\mathfrak{g}^{*}$ a Lie algebra.

(b) The cobracket $\psi: \mathfrak{g} \longrightarrow \mathfrak{g} \wedge \mathfrak{g}$ is a 1-cocycle on $\mathfrak{g}$ with respect to the $\mathfrak{g}$-module structure on $\mathfrak{g} \wedge \mathfrak{g}$ given by the adjoint action. In other words, we have that for any $a, b \in \mathfrak{g}$,

$$
\psi([a, b])=a \cdot \psi(b)-b \cdot \psi(a),
$$

where

$$
a \cdot(b \otimes c)=[a \otimes 1+1 \otimes a, b \otimes c]=[a, b] \otimes c+b \otimes[a, c] .
$$

In a Lie bialgebra $(\mathfrak{g}, \psi)$, a Lie ideal $\mathfrak{b}$ of $\mathfrak{g}$ is said to be a Lie bialgebra ideal if $\psi(\mathfrak{b}) \subseteq \mathfrak{g} \otimes \mathfrak{b}+\mathfrak{b} \otimes \mathfrak{g}$. A Lie homomorphism $\varphi:(\mathfrak{g}, \psi) \longrightarrow\left(\mathfrak{g}^{\prime}, \psi^{\prime}\right)$ is said to be a Lie bialgebra homomorphism if $(\varphi \otimes \varphi) \circ \psi=\psi^{\prime} \circ \varphi$. Note that if $\mathfrak{b}$ is a Lie bialgebra ideal of $(\mathfrak{g}, \psi)$, then $(\mathfrak{g} / \mathfrak{b}, \bar{\psi})$ is also a Lie bialgebra. A Lie bialgebra $(\mathfrak{g}, \psi)$ is frequently denoted by $\left(\mathfrak{g}, \mathfrak{g}^{*}\right)$.

Theorem. Let $(A, \iota, m,\{\cdot, \cdot\}, \epsilon, \Delta)$ be a Poisson bialgebra and let $\mathfrak{m}=\operatorname{ker} \epsilon$. Then $\left(\left(\mathfrak{m} / \mathfrak{m}^{2}\right)^{*}, \mathfrak{m} / \mathfrak{m}^{2}\right)$ is a Lie bialgebra.

Proof. We will show that the pair $\left(\left(\mathfrak{m} / \mathfrak{m}^{2}\right)^{*}, \psi\right)$ is a Lie bialgebra, where $\psi$ : $\left(\mathfrak{m} / \mathfrak{m}^{2}\right)^{*} \longrightarrow\left(\mathfrak{m} / \mathfrak{m}^{2}\right)^{*} \wedge\left(\mathfrak{m} / \mathfrak{m}^{2}\right)^{*}$ is defined by

$$
\psi(f)\left(z_{1} \otimes z_{2}\right)=f\left(\left[z_{1}, z_{2}\right]\right)
$$

for all $z_{1}, z_{2} \in \mathfrak{m} / \mathfrak{m}^{2}$. It is enough to prove that $\psi$ is a 1 -cocycle on $\left(\mathfrak{m} / \mathfrak{m}^{2}\right)^{*}$. The natural $\mathbf{k}$-bilinear form $\langle\cdot, \cdot\rangle$ defined by

$$
\langle\cdot, \cdot\rangle:\left(\mathfrak{m} / \mathfrak{m}^{2}\right)^{*} \times \mathfrak{m} / \mathfrak{m}^{2} \longrightarrow \mathbf{k},\left\langle f, a+\mathfrak{m}^{2}\right\rangle=f\left(a+\mathfrak{m}^{2}\right)
$$


is a nondegenerate $\mathbf{k}$-bilinear form. Identifying $\left(\mathfrak{m} / \mathfrak{m}^{2}\right)^{*}$ to $P_{\epsilon}\left(A^{\circ}\right)$ by 1.4 , we have that, for $f, g \in\left(\mathfrak{m} / \mathfrak{m}^{2}\right)^{*}$ and $a, b \in \mathfrak{m}$,

$$
\begin{aligned}
\left\langle\psi([f, g]),\left(a+\mathfrak{m}^{2}\right) \otimes\left(b+\mathfrak{m}^{2}\right)\right\rangle= & \left\langle[f, g],\{a, b\}+\mathfrak{m}^{2}\right\rangle \\
= & (f g)(\{a, b\})-(g f)(\{a, b\}) \\
= & \sum f\left(a^{\prime} b^{\prime}\right) g\left(\left\{a^{\prime \prime}, b^{\prime \prime}\right\}\right)+f\left(\left\{a^{\prime}, b^{\prime}\right\}\right) g\left(a^{\prime \prime} b^{\prime \prime}\right) \\
& -g\left(a^{\prime} b^{\prime}\right) f\left(\left\{a^{\prime \prime}, b^{\prime \prime}\right\}\right)-g\left(\left\{a^{\prime}, b^{\prime}\right\}\right) f\left(a^{\prime \prime} b^{\prime \prime}\right) \\
= & \sum\left(\epsilon\left(a^{\prime}\right) f\left(b^{\prime}\right)+f\left(a^{\prime}\right) \epsilon\left(b^{\prime}\right)\right) g\left(\left\{a^{\prime \prime}, b^{\prime \prime}\right\}\right) \\
& +f\left(\left\{a^{\prime}, b^{\prime}\right\}\right)\left(\epsilon\left(a^{\prime \prime}\right) g\left(b^{\prime \prime}\right)+g\left(a^{\prime \prime}\right) \epsilon\left(b^{\prime \prime}\right)\right) \\
& -\left(\epsilon\left(a^{\prime}\right) g\left(b^{\prime}\right)+g\left(a^{\prime}\right) \epsilon\left(b^{\prime}\right)\right) f\left(\left\{a^{\prime \prime}, b^{\prime \prime}\right\}\right) \\
& -g\left(\left\{a^{\prime}, b^{\prime}\right\}\right)\left(\epsilon\left(a^{\prime \prime}\right) f\left(b^{\prime \prime}\right)+f\left(a^{\prime \prime}\right) \epsilon\left(b^{\prime \prime}\right)\right) \\
= & \sum f\left(b^{\prime}\right) g\left(\left\{a, b^{\prime \prime}\right\}\right)+f\left(a^{\prime}\right) g\left(\left\{a^{\prime \prime}, b\right\}\right) \\
& +f\left(\left\{a^{\prime}, b\right\}\right) g\left(a^{\prime \prime}\right)+f\left(\left\{a, b^{\prime}\right\}\right) g\left(b^{\prime \prime}\right) \\
& -g\left(b^{\prime}\right) f\left(\left\{a, b^{\prime \prime}\right\}\right)-g\left(a^{\prime}\right) f\left(\left\{a^{\prime \prime}, b\right\}\right) \\
& -g\left(\left\{a, b^{\prime}\right\}\right) f\left(b^{\prime \prime}\right)-g\left(\left\{a^{\prime}, b\right\}\right) f\left(a^{\prime \prime}\right) \\
&
\end{aligned}
$$

by (2). Let

$$
\psi(f)=\sum f_{1} \otimes f_{2}, \quad \psi(g)=\sum g_{1} \otimes g_{2} .
$$

Then, by (4), we have

$$
\begin{aligned}
& f(\{a, b\})=\left\langle\psi(f),\left(a+\mathfrak{m}^{2}\right) \otimes\left(b+\mathfrak{m}^{2}\right)\right\rangle=\sum f_{1}(a) f_{2}(b), \\
& g(\{a, b\})=\left\langle\psi(g),\left(a+\mathfrak{m}^{2}\right) \otimes\left(b+\mathfrak{m}^{2}\right)\right\rangle=\sum g_{1}(a) g_{2}(b)
\end{aligned}
$$

for all $a, b \in \mathfrak{m}$. Hence

$$
\begin{aligned}
& \left\langle f \cdot \psi(g)-g \cdot \psi(f),\left(a+\mathfrak{m}^{2}\right) \otimes\left(b+\mathfrak{m}^{2}\right)\right\rangle \\
= & \left\langle\sum\left[f, g_{1}\right] \otimes g_{2}+g_{1} \otimes\left[f, g_{2}\right]-\left[g, f_{1}\right] \otimes f_{2}-f_{1} \otimes\left[g, f_{2}\right],\right. \\
& \left.\left(a+\mathfrak{m}^{2}\right) \otimes\left(b+\mathfrak{m}^{2}\right)\right\rangle \\
= & \sum f\left(a^{\prime}\right) g_{1}\left(a^{\prime \prime}\right) g_{2}(b)-g_{1}\left(a^{\prime}\right) f\left(a^{\prime \prime}\right) g_{2}(b) \\
& +g_{1}(a) f\left(b^{\prime}\right) g_{2}\left(b^{\prime \prime}\right)-g_{1}(a) g_{2}\left(b^{\prime}\right) f\left(b^{\prime \prime}\right) \\
& -g\left(a^{\prime}\right) f_{1}\left(a^{\prime \prime}\right) f_{2}(b)+f_{1}\left(a^{\prime}\right) g\left(a^{\prime \prime}\right) f_{2}(b) \\
& -f_{1}(a) g\left(b^{\prime}\right) f_{2}\left(b^{\prime \prime}\right)+f_{1}(a) f_{2}\left(b^{\prime}\right) g\left(b^{\prime \prime}\right) \\
= & \sum f\left(a^{\prime}\right) g\left(\left\{a^{\prime \prime}, b\right\}\right)-g\left(\left\{a^{\prime}, b\right\}\right) f\left(a^{\prime \prime}\right) \\
& +f\left(b^{\prime}\right) g\left(\left\{a, b^{\prime \prime}\right\}\right)-g\left(\left\{a, b^{\prime}\right\}\right) f\left(b^{\prime \prime}\right) \\
& -g\left(a^{\prime}\right) f\left(\left\{a^{\prime \prime}, b\right\}\right)+f\left(\left\{a^{\prime}, b\right\}\right) g\left(a^{\prime \prime}\right) \\
& -g\left(b^{\prime}\right) f\left(\left\{a, b^{\prime \prime}\right\}\right)+f\left(\left\{a, b^{\prime}\right\}\right) g\left(b^{\prime \prime}\right) .
\end{aligned}
$$


Thus we have $\psi([f, g])=f \cdot \psi(g)-g \cdot \psi(f)$ for all $f, g \in\left(\mathfrak{m} / \mathfrak{m}^{2}\right)^{*}$ and so $\psi$ is a 1-cocycle as claimed.

Example 1.6. Let $q$ be an indeterminate over k. By [1, I.2.2], the coordinate ring of quantum $n \times n$-matrices, denoted by $\mathcal{O}_{q}\left(M_{n}(\mathbf{k})\right)$, is the $\mathbf{k}\left[q^{ \pm 1}\right]$-algebra generated by $x_{i j}, 1 \leq i, j \leq n$, subject to the relations

$$
x_{i j} x_{r s}= \begin{cases}q x_{r s} x_{i j} & i=r \text { and } j<s, \\ q x_{r s} x_{i j} & i<r \text { and } j=s, \\ x_{r s} x_{i j} & i<r \text { and } j>s, \\ x_{r s} x_{i j}+\left(q-q^{-1}\right) x_{i s} x_{r j} & i<r \text { and } j<s .\end{cases}
$$

Thus

$$
x_{i j} x_{r s}-x_{r s} x_{i j}= \begin{cases}(q-1) x_{r s} x_{i j} & i=r \text { and } j<s, \\ (q-1) x_{r s} x_{i j} & i<r \text { and } j=s, \\ 0 & i<r \text { and } j>s, \\ q^{-1}(q-1)(q+1) x_{i s} x_{r j} & i<r \text { and } j<s .\end{cases}
$$

Hence $\mathcal{O}_{q}\left(M_{n}(\mathbf{k})\right) /\langle q-1\rangle$ is the commutative $\mathbf{k}$-algebra $\mathbf{k}\left[\bar{x}_{i j} \mid i, j=1, \ldots, n\right]$. Moreover $\mathcal{O}_{q}\left(M_{n}(\mathbf{k})\right) /\langle q-1\rangle$ is a Poisson algebra with Poisson bracket

$$
\left\{\bar{x}_{i j}, \bar{x}_{r s}\right\}=\overline{(q-1)^{-1}\left(x_{i j} x_{r s}-x_{r s} x_{i j}\right)}
$$

by [1, III.5.4]. More precisely, we have that

$$
\left\{\bar{x}_{i j}, \bar{x}_{r s}\right\}= \begin{cases}\bar{x}_{r s} \bar{x}_{i j} & i=r \text { and } j<s, \\ \bar{x}_{r s} \bar{x}_{i j} & i<r \text { and } j=s, \\ 0 & i<r \text { and } j>s, \\ 2 \bar{x}_{i s} \bar{x}_{r j} & i<r \text { and } j<s .\end{cases}
$$

The coordinate ring of $n \times n$-matrices is the commutative $\mathbf{k}$-algebra

$$
\mathbf{k}\left[x_{i j} \mid i, j=1, \ldots, n\right],
$$

denoted by $\mathcal{O}\left(M_{n}(\mathbf{k})\right)$, which is a bialgebra with the coalgebra structure

$$
\epsilon\left(x_{i j}\right)=\delta_{i j}, \quad \Delta\left(x_{i j}\right)=\sum_{k=1}^{n} x_{i k} \otimes x_{k j} .
$$

The algebra $\mathcal{O}\left(M_{n}(\mathbf{k})\right)$ is also a Poisson algebra with Poisson bracket

$$
\left\{x_{i j}, x_{r s}\right\}= \begin{cases}x_{i j} x_{r s} & i=r \text { and } j<s \\ x_{i j} x_{r s} & i<r \text { and } j=s \\ 0 & i<r \text { and } j>s \\ 2 x_{i s} x_{r j} & i<r \text { and } j<s\end{cases}
$$

by the above paragraph. Moreover $\mathcal{O}\left(M_{n}(\mathbf{k})\right)$ is a Poisson bialgebra since

$$
\Delta\left(\left\{x_{i j}, x_{r s}\right\}\right)=\left\{\Delta\left(x_{i j}\right), \Delta\left(x_{r s}\right)\right\}
$$

for all $i, j, r, s$, any Poisson bracket satisfies the Leibniz rule and $\Delta$ is an algebra homomorphism. 
In $\mathfrak{m} / \mathfrak{m}^{2}$, set

$$
e_{i j}=x_{i j}+\mathfrak{m}^{2}, \quad e_{k k}=\left(x_{k k}-1\right)+\mathfrak{m}^{2}, \quad i \neq j, 1 \leq k \leq n .
$$

Then $e_{i j}, i, j=1, \ldots, n$, form a $\mathbf{k}$-basis of $\mathfrak{m} / \mathfrak{m}^{2}$ and satisfy

$$
\begin{aligned}
& {\left[e_{i i}, e_{i s}\right]=e_{i s} \quad i<s,} \\
& {\left[e_{i i}, e_{i s}\right]=-e_{i s} \quad i>s,} \\
& {\left[e_{i j}, e_{i s}\right]=0 \quad i \neq j, i \neq s,} \\
& {\left[e_{i i}, e_{r i}\right]=e_{r i} \quad i<r,} \\
& {\left[e_{i i}, e_{r i}\right]=-e_{r i} \quad i>r,} \\
& {\left[e_{i j}, e_{r j}\right]=0 \quad i \neq j, r \neq j,} \\
& {\left[e_{i j}, e_{r s}\right]=0 \quad i<r, j<s, i \neq s, r \neq j,} \\
& {\left[e_{i j}, e_{r s}\right]=2 e_{r j} \quad i<r, j<s, i=s, r \neq j,} \\
& {\left[e_{i j}, e_{r s}\right]=2 e_{i s} \quad i<r, j<s, i \neq s, r=j \text {, }} \\
& {\left[e_{i j}, e_{r s}\right]=0 \quad i<r, j>s,} \\
& {\left[e_{i i}, e_{r r}\right]=0 \quad i \neq r}
\end{aligned}
$$

by (5). The dual $\left(\mathfrak{m} / \mathfrak{m}^{2}\right)^{*}$ has the dual basis $e_{i j}^{*}$ for $e_{i j}, i, j=1, \ldots, n$, satisfying

$$
\left[e_{i j}^{*}, e_{r s}^{*}\right]=\delta_{j r} e_{i s}^{*}-\delta_{s i} e_{r j}^{*}
$$

for all $i, j, r, s$. That is, $\left(\mathfrak{m} / \mathfrak{m}^{2}\right)^{*}$ is isomorphic to the general linear Lie algebra $\mathfrak{g l}_{n}(\mathbf{k})$. Moreover the pair $\left(\left(\mathfrak{m} / \mathfrak{m}^{2}\right)^{*}, \mathfrak{m} / \mathfrak{m}^{2}\right)$ is a Lie bialgebra by 1.5 . Now the cobracket $\psi:\left(\mathfrak{m} / \mathfrak{m}^{2}\right)^{*} \longrightarrow\left(\mathfrak{m} / \mathfrak{m}^{2}\right)^{*} \wedge\left(\mathfrak{m} / \mathfrak{m}^{2}\right)^{*}$ is given by

$$
\begin{array}{ll}
\psi\left(e_{i i}^{*}\right)=0 & i=1, \ldots, n, \\
\psi\left(e_{i j}^{*}\right)=e_{i i}^{*} \wedge e_{i j}^{*}+e_{i j}^{*} \wedge e_{j j}^{*}+\sum_{i<k<j} 2 e_{i k}^{*} \wedge e_{k j}^{*} & i<j, \\
\psi\left(e_{i j}^{*}\right)=e_{i j}^{*} \wedge e_{i i}^{*}+e_{j j}^{*} \wedge e_{i j}^{*}+\sum_{j<k<i} 2 e_{k j}^{*} \wedge e_{i k}^{*} & i>j .
\end{array}
$$

Example 1.7. Let $\mathfrak{b}$ denote the Lie ideal $\mathbf{k}\left(\sum_{i} e_{i i}^{*}\right)$ of $\left(\mathfrak{m} / \mathfrak{m}^{2}\right)^{*}$ in Example 1.6. Then $\mathfrak{b}$ is a Lie bialgebra ideal since $\psi(\mathfrak{b}) \subseteq\left(\mathfrak{m} / \mathfrak{m}^{2}\right)^{*} \otimes \mathfrak{b}+\mathfrak{b} \otimes\left(\mathfrak{m} / \mathfrak{m}^{2}\right)^{*}$ and thus $\left(\mathfrak{m} / \mathfrak{m}^{2}\right)^{*} / \mathfrak{b}$ is also a Lie bialgebra. In fact, it is checked immediately that the Lie bialgebra $\left(\mathfrak{m} / \mathfrak{m}^{2}\right)^{*} / \mathfrak{b}$ is isomorphic to the well-known Lie bialgebra $\left(\mathfrak{s l}_{n}(\mathbf{k}), \delta\right)$, where $\delta: \mathfrak{s l}_{n}(\mathbf{k}) \longrightarrow \mathfrak{s l}_{n}(\mathbf{k}) \wedge \mathfrak{s l}_{n}(\mathbf{k})$ is given by

$$
\delta\left(h_{i}\right)=0, \quad \delta\left(E_{i, i+1}\right)=h_{i} \wedge E_{i, i+1}, \quad \delta\left(E_{i+1, i}\right)=h_{i} \wedge E_{i+1, i},
$$

where $E_{i j}$ is the $n \times n$-matrix with 0 for all positions except $(i, j)$-position and 1 for $(i, j)$-position and $h_{i}=E_{i i}-E_{i+1, i+1}$ for $i=1, \ldots, n-1$ (The cobracket $\delta$ is uniquely determined by (6) since $\delta$ is a 1-cocycle and $\mathfrak{s l}_{n}(\mathbf{k})$ is generated by $\left.h_{i}, E_{i, i+1}, E_{i+1, i}, i=1, \ldots, n-1\right)$. The cobracket $\delta$ in $(6)$ is the standard Lie bialgebra structure in $\mathfrak{s l}_{n}(\mathbf{k})$ (see $[2,1.3 .8]$ ). 


\section{Application}

2.1. Let $A=(A, \iota, m, \epsilon, \Delta)$ be a bialgebra. Note that the dual $A^{*}$ is an $A$ - $A$ bimodule:

$$
(a \varphi b)(x)=\varphi(b x a), \quad \varphi \in A^{*}, a, b, x \in A .
$$

For a left $A$-module $M$, the dual space $M^{*}$ is a right $A$-module with structure

$$
(f a)(x)=f(a x), \quad a \in A, f \in M^{*}, x \in M .
$$

Let $\mathcal{C}$ be a class of finite dimensional left $A$-modules which is closed under finite direct sums and finite tensor products. For any $M \in \mathcal{C}, f \in M^{*}$ and $v \in M$, the coordinate function $c_{f, v}^{M} \in A^{*}$ is defined by

$$
c_{f, v}^{M}(x)=f(x v), \quad x \in A .
$$

Then $c_{f, v}^{M}$ is an element of the restricted dual $A^{\circ}$ of $A$ since the annihilator $I$ of $M$ is an ideal of $A$ such that the dimension of $A / I$ is finite and $c_{f, v}^{M}(I)=0$. It is well-known that the vector space $A(\mathcal{C})$ spanned by all coordinate functions $c_{f, v}^{M}, M \in \mathcal{C}, f \in M^{*}, v \in M$, is a sub-bialgebra of $A^{\circ}$ with structure

$$
\begin{array}{ll}
c_{f, v}^{M}+c_{g, w}^{N}=c_{(f, g),(v, w)}^{M \oplus N}, & c_{f, v}^{M} c_{g, w}^{N}=c_{f \otimes g, v \otimes w}^{M \otimes N}, \\
\Delta\left(c_{f, v}^{M}\right)=\sum_{i} c_{f, v_{i}}^{M} \otimes c_{f_{i}, v}^{M}, & \epsilon\left(c_{f, v}^{M}\right)=f(v),
\end{array}
$$

where $\left\{v_{i}\right\}$ and $\left\{f_{i}\right\}$ are dual bases for $M$ and $M^{*}$ (see [1, I.7]). Moreover if $A$ is a Hopf algebra and $\mathcal{C}$ is closed under duals, then $A(\mathcal{C})$ is a Hopf algebra with antipode $S$ defined by

$$
S\left(c_{f, v}^{M}\right)=c_{v, f}^{M^{*}}, \quad M \in \mathcal{C}, f \in M^{*}, v \in M .
$$

Observe that $A(\mathcal{C})$ has a left and right $A$-action induced by $(7)$ :

$$
a \cdot c_{f, v}^{M}=c_{f, a v}^{M}, \quad c_{f, v}^{M} \cdot a=c_{f a, v}^{M}, \quad a \in A .
$$

2.2. Let $(\mathfrak{g}, \psi)$ be a Lie bialgebra and let $\Delta$ be the comultiplication of $U(\mathfrak{g})$. The cobracket $\psi$ is extended uniquely to a $\Delta$-derivation $\bar{\psi}$ from $U(\mathfrak{g})$ into $U(\mathfrak{g}) \otimes U(\mathfrak{g})$. That is,

$$
\bar{\psi}: U(\mathfrak{g}) \longrightarrow U(\mathfrak{g}) \otimes U(\mathfrak{g})
$$

is a $\mathbf{k}$-linear map such that $\left.\bar{\psi}\right|_{\mathfrak{g}}=\psi$ and $\bar{\psi}(x y)=\bar{\psi}(x) \Delta(y)+\Delta(x) \bar{\psi}(y)$ for all $x, y \in U(\mathfrak{g})$.

Let $(\mathfrak{g}, \psi)$ be a coboundary Lie bialgebra such that the cobracket $\psi$ determined by a classical $r$-matrix $r=\sum_{i} a_{i} \otimes b_{i}$. That is, $r$ satisfies the modified classical Yang-Baxter equation and $\psi$ is defined by

$$
\psi(x)=x \cdot r=\sum_{i}\left[x, a_{i}\right] \otimes b_{i}+a_{i} \otimes\left[x, b_{i}\right]=[\Delta(x), r]_{U(\mathfrak{g}) \otimes U(\mathfrak{g})}
$$


for all $x \in \mathfrak{g}$ (refer to $[2,2.1]$ and $[9, \S 4.1]$ for the definition of a coboundary Lie bialgebra). Then the extension map $\bar{\psi}$ of $\psi$ to $U(\mathfrak{g})$ is given by $\bar{\psi}(x)=$ $[\Delta(x), r]_{U(\mathfrak{g}) \otimes U(\mathfrak{g})}$ for all $x \in U(\mathfrak{g})$.

Theorem. Let $(\mathfrak{g}, \psi)$ be a coboundary Lie bialgebra such that the cobracket $\psi$ is determined by a classical $r$-matrix $r$. Fix a class $\mathcal{C}$ of finite dimensional left $U(\mathfrak{g})$-modules which is closed under finite direct sums and finite tensor products. Denote by $A(\mathcal{C})$ the vector space spanned by all coordinate functions $c_{f, v}^{M}, M \in \mathcal{C}, f \in M^{*}, v \in M$. Then $A(\mathcal{C})$ is a Poisson bialgebra with Poisson bracket

$$
\left\{c_{f, v}^{M}, c_{g, w}^{N}\right\}(x)=\left\langle\bar{\psi}(x), c_{f, v}^{M} \otimes c_{g, w}^{N}\right\rangle
$$

for all $x \in U(\mathfrak{g})$.

Remark. Observe that, in the above theorem, $A(\mathcal{C})$ is a sub-Poisson bialgebra of the restricted dual $U(\mathfrak{g})^{\circ}$ and we obtain a Lie bialgebra $\left(\left(\mathfrak{m} / \mathfrak{m}^{2}\right)^{*}, \mathfrak{m} / \mathfrak{m}^{2}\right)$ by applying 1.5 to $A(\mathcal{C})$, where $\mathfrak{m}$ is the kernel of the counit in $A(\mathcal{C})$.

Proof of Theorem. We have already known that $A(\mathcal{C})$ is a sub-bialgebra of the restricted dual $U(\mathfrak{g})^{\circ}$ with structure (8) by 2.1 .

Denote $r=\sum_{i} a_{i} \otimes b_{i}$. Then

$$
\bar{\psi}(x)=[\Delta(x), r]_{U(\mathfrak{g}) \otimes U(\mathfrak{g})}=\sum_{(x)} \sum_{i}\left(x^{\prime} a_{i} \otimes x^{\prime \prime} b_{i}-a_{i} x^{\prime} \otimes b_{i} x^{\prime \prime}\right)
$$

for all $x \in U(\mathfrak{g})$, thus

$$
\begin{aligned}
\left\{c_{f, v}^{M}, c_{g, w}^{N}\right\}(x) & =\sum_{i} \sum_{(x)} c_{f, v}^{M}\left(x^{\prime} a_{i}\right) c_{g, w}^{N}\left(x^{\prime \prime} b_{i}\right)-\sum_{i} \sum_{(x)} c_{f, v}^{M}\left(a_{i} x^{\prime}\right) c_{g, w}^{N}\left(b_{i} x^{\prime \prime}\right) \\
& =\sum_{i}\left(c_{f, a_{i} v}^{M} c_{g, b_{i} w}^{N}\right)(x)-\sum_{i}\left(c_{f a_{i}, v}^{M} c_{g b_{i}, w}^{N}\right)(x)
\end{aligned}
$$

Hence

$$
\left\{c_{f, v}^{M}, c_{g, w}^{N}\right\}=\sum_{i}\left(c_{f, a_{i} v}^{M} c_{g, b_{i} w}^{N}\right)-\sum_{i}\left(c_{f a_{i}, v}^{M} c_{g b_{i}, w}^{N}\right) \in A(\mathcal{C})
$$

that is, the Poisson bracket $(10)$ is well-defined.

Let $\tau: U(\mathfrak{g}) \otimes U(\mathfrak{g}) \longrightarrow U(\mathfrak{g}) \otimes U(\mathfrak{g})$ be the flip. Since $U(\mathfrak{g})$ is cocommutative and $\tau(r)=-r$, we have that $\tau \bar{\psi}(x)=-\bar{\psi}(x)$ for all $x \in U(\mathfrak{g})$, thus we have immediately that $\left\{c_{f, v}^{M}, c_{g, w}^{N}\right\}=-\left\{c_{g, w}^{N}, c_{f, v}^{M}\right\}$ for all $c_{f, v}^{M}, c_{g, w}^{N} \in A(\mathcal{C})$ by $(10)$.

For distinct numbers $s, t=1,2,3$, denote by $r_{s t} \in \mathfrak{g} \otimes \mathfrak{g} \otimes \mathfrak{g}$ the element with $a_{i}$ for $s$-component, $b_{i}$ for $t$-component and 1 for the other component. For instance, $r_{12}=\sum a_{i} \otimes b_{i} \otimes 1$ and $r_{31}=\sum b_{i} \otimes 1 \otimes a_{i}$. Note that $r_{s t}=-r_{t s}$ for all distinct numbers $s, t=1,2,3$, by the skew symmetry of $r$. Since $\Delta(a)=$ 
$a \otimes 1+1 \otimes a$ for all $a \in \mathfrak{g}$, we have

$$
\begin{aligned}
\left\{\left\{c_{f, v}^{M}, c_{g, w}^{N}\right\}, c_{h, u}^{L}\right\}(x)= & \left\langle\Delta^{2}(x)\left(r_{13}+r_{23}\right) r_{12}, c_{f, v}^{M} \otimes c_{g, w}^{N} \otimes c_{h, u}^{L}\right\rangle \\
& -\left\langle r_{12} \Delta^{2}(x)\left(r_{13}+r_{23}\right), c_{f, v}^{M} \otimes c_{g, w}^{N} \otimes c_{h, u}^{L}\right\rangle \\
& -\left\langle\left(r_{13}+r_{23}\right) \Delta^{2}(x) r_{12}, c_{f, v}^{M} \otimes c_{g, w}^{N} \otimes c_{h, u}^{L}\right\rangle \\
& +\left\langle r_{12}\left(r_{13}+r_{23}\right) \Delta^{2}(x), c_{f, v}^{M} \otimes c_{g, w}^{N} \otimes c_{h, u}^{L}\right\rangle
\end{aligned}
$$

for $x \in U(\mathfrak{g})$, where $\Delta^{2}=(\Delta \otimes 1) \circ \Delta=(1 \otimes \Delta) \circ \Delta$, by (10). Hence, by $r_{s t}=-r_{t s}$ for all $s, t=1,2,3$ and the coassociativity of $\Delta$, we have that

$$
\begin{aligned}
& \left(\left\{\left\{c_{f, v}^{M}, c_{g, w}^{N}\right\}, c_{h, u}^{L}\right\}+\left\{\left\{c_{g, w}^{N}, c_{h, u}^{L}\right\}, c_{f, v}^{M}\right\}+\left\{\left\{c_{h, u}^{L}, c_{f, v}^{M}\right\}, c_{g, w}^{N}\right\}\right)(x) \\
= & \left\langle\Delta^{2}(x)\left(r_{13}+r_{23}\right) r_{12}, c_{f, v}^{M} \otimes c_{g, w}^{N} \otimes c_{h, u}^{L}\right\rangle \\
& -\left\langle r_{12} \Delta^{2}(x)\left(r_{13}+r_{23}\right), c_{f, v}^{M} \otimes c_{g, w}^{N} \otimes c_{h, u}^{L}\right\rangle \\
& -\left\langle\left(r_{13}+r_{23}\right) \Delta^{2}(x) r_{12}, c_{f, v}^{M} \otimes c_{g, w}^{N} \otimes c_{h, u}^{L}\right\rangle \\
& +\left\langle r_{12}\left(r_{13}+r_{23}\right) \Delta^{2}(x), c_{f, v}^{M} \otimes c_{g, w}^{N} \otimes c_{h, u}^{L}\right\rangle \\
& +\left\langle\Delta^{2}(x)\left(r_{21}+r_{31}\right) r_{23}, c_{f, v}^{M} \otimes c_{g, w}^{N} \otimes c_{h, u}^{L}\right\rangle \\
& -\left\langle r_{23} \Delta^{2}(x)\left(r_{21}+r_{31}\right), c_{f, v}^{M} \otimes c_{g, w}^{N} \otimes c_{h, u}^{L}\right\rangle \\
& -\left\langle\left(r_{21}+r_{31}\right) \Delta^{2}(x) r_{23}, c_{f, v}^{M} \otimes c_{g, w}^{N} \otimes c_{h, u}^{L}\right\rangle \\
& +\left\langle r_{23}\left(r_{21}+r_{31}\right) \Delta^{2}(x), c_{f, v}^{M} \otimes c_{g, w}^{N} \otimes c_{h, u}^{L}\right\rangle \\
& +\left\langle\Delta^{2}(x)\left(r_{32}+r_{12}\right) r_{31}, c_{f, v}^{M} \otimes c_{g, w}^{N} \otimes c_{h, u}^{L}\right\rangle \\
& -\left\langle r_{31} \Delta^{2}(x)\left(r_{32}+r_{12}\right), c_{f, v}^{M} \otimes c_{g, w}^{N} \otimes c_{h, u}^{L}\right\rangle \\
& -\left\langle\left(r_{32}+r_{12}\right) \Delta^{2}(x) r_{31}, c_{f, v}^{M} \otimes c_{g, w}^{N} \otimes c_{h, u}^{L}\right\rangle \\
& +\left\langle r_{31}\left(r_{32}+r_{12}\right) \Delta^{2}(x), c_{f, v}^{M} \otimes c_{g, w}^{N} \otimes c_{h, u}^{L}\right\rangle \\
= & \left.\left\langle\left(\left[r_{12}, r_{13}\right]+\left[r_{12}, r_{23}\right]+\left[r_{13}, r_{23}\right]\right) \Delta^{2}(x), c_{f, v}^{M} \otimes c_{g, w}^{N} \otimes c_{h, u}^{L}\right)\right\rangle \\
& \left.-\left\langle\Delta^{2}(x)\left(\left[r_{12}, r_{13}\right]+\left[r_{12}, r_{23}\right]+\left[r_{13}, r_{23}\right]\right), c_{f, v}^{M} \otimes c_{g, w}^{N} \otimes c_{h, u}^{L}\right)\right\rangle \\
= & 0
\end{aligned}
$$

for any $c_{f, v}^{M}, c_{g, w}^{N}, c_{h, u}^{L} \in A(\mathcal{C})$ and $x \in U(\mathfrak{g})$ since

$$
\begin{aligned}
& {\left[r_{12}, r_{13}\right]+\left[r_{12}, r_{23}\right]+\left[r_{13}, r_{23}\right] } \\
= & \left(r_{12} r_{13}-r_{13} r_{12}\right)+\left(r_{12} r_{23}-r_{23} r_{12}\right)+\left(r_{13} r_{23}-r_{23} r_{13}\right) \\
= & \sum_{i, j}\left[a_{i}, a_{j}\right] \otimes b_{i} \otimes b_{j}+\sum_{i, j} a_{i} \otimes\left[b_{i}, a_{j}\right] \otimes b_{j}+\sum_{i, j} a_{i} \otimes a_{j} \otimes\left[b_{i}, b_{j}\right]
\end{aligned}
$$

is $\mathfrak{g}$-invariant. Hence the Poisson bracket (10) satisfies the Jacobi identity. 
By (11), we have

$$
\begin{aligned}
\left\{c_{f, v}^{M}, c_{g, w}^{N} c_{h, u}^{L}\right\}= & \sum_{i} c_{f, a_{i} v}^{M}\left(c_{g, b_{i} w}^{N} c_{h, u}^{L}+c_{g, w}^{N} c_{h, b_{i} u}^{L}\right) \\
& -\sum_{i} c_{f a_{i}, v}^{M}\left(c_{g b_{i}, w}^{N} c_{h, u}^{L}+c_{g, w}^{N} c_{h b_{i}, u}^{L}\right) \\
= & \left\{c_{f, v}^{M}, c_{g, w}^{N}\right\} c_{h, u}^{L}+c_{g, w}^{N}\left\{c_{f, v}^{M}, c_{h, u}^{L}\right\} .
\end{aligned}
$$

It follows that the Poisson bracket (10) satisfies the Leibniz rule.

Let us prove that $\Delta\left(\left\{c_{f, v}^{M}, c_{g, w}^{N}\right\}\right)=\left\{\Delta\left(c_{f, v}^{M}\right), \Delta\left(c_{g, w}^{N}\right)\right\}$ for all elements $c_{f, v}^{M}$, $c_{g, w}^{N} \in A(\mathcal{C})$. Note that $\Delta\left(c_{f, v}^{M}\right)=\sum_{j} c_{f, v_{j}}^{M} \otimes c_{f_{j}, v}^{M}, \Delta\left(c_{g, w}^{N}\right)=\sum_{k} c_{g, w_{k}}^{N} \otimes c_{g_{k}, w}^{N}$, where $\left\{v_{j}\right\},\left\{f_{j}\right\}$ are dual bases for $M$ and $M^{*}$ and $\left\{w_{k}\right\},\left\{g_{k}\right\}$ are dual bases for $N$ and $N^{*}$. Now, for any $x, y \in U(\mathfrak{g})$,

$$
\begin{aligned}
& \Delta\left(\left\{c_{f, v}^{M}, c_{g, w}^{N}\right\}\right)(x \otimes y)=\left\langle\bar{\psi}(x y), c_{f, v}^{M} \otimes c_{g, w}^{N}\right\rangle \\
&=\left\langle\bar{\psi}(x) \Delta(y), c_{f, v}^{M} \otimes c_{g, w}^{N}\right\rangle+\left\langle\Delta(x) \bar{\psi}(y), c_{f, v}^{M} \otimes c_{g, w}^{N}\right\rangle \\
&= \sum_{j, k}\left\langle\bar{\psi}(x), c_{f, v_{j}}^{M} \otimes c_{g, w_{k}}^{N}\right\rangle\left\langle\Delta(y), c_{f_{j}, v}^{M} \otimes c_{g_{k}, w}^{N}\right\rangle \\
& \quad+\sum_{j, k}\left\langle\Delta(x), c_{f, v_{j}}^{M} \otimes c_{g, w_{k}}^{N}\right\rangle\left\langle\bar{\psi}(y), c_{f_{j}, v}^{M} \otimes c_{g_{k}, w}^{N}\right\rangle \\
&= \sum_{j, k}\left(\left\{c_{f, v_{j}}^{M}, c_{g, w_{k}}^{N}\right\} \otimes c_{f_{j}, v}^{M} c_{g_{k}, w}^{N}\right)(x \otimes y) \\
& \quad+\sum_{j, k}\left(c_{f, v_{j}}^{M} c_{g, w_{k}}^{N} \otimes\left\{c_{f_{j}, v}^{M}, c_{g_{k}, w}^{N}\right\}\right)(x \otimes y) \\
&=\left\{\Delta\left(c_{f, v}^{M}\right), \Delta\left(c_{g, w}^{N}\right)\right\}(x \otimes y) .
\end{aligned}
$$

Hence we have $\Delta\left(\left\{c_{f, v}^{M}, c_{g, w}^{N}\right\}\right)=\left\{\Delta\left(c_{f, v}^{M}\right), \Delta\left(c_{g, w}^{N}\right)\right\}$ for all elements $c_{f, v}^{M}, c_{g, w}^{N} \in$ $A(\mathcal{C})$. This completes the proof.

Proposition 2.3. Let $(\mathfrak{g}, \psi)$ be a coboundary Lie bialgebra such that $\mathfrak{g}$ is connected and simply connected and let $\mathcal{C}$ be the set of all finite dimensional left $U(\mathfrak{g})$-modules. Then $A(\mathcal{C})$ is the restricted dual $U(\mathfrak{g})^{\circ}$. Moreover the given Lie bialgebra $(\mathfrak{g}, \psi)$ is isomorphic to $\left(\left(\mathfrak{m} / \mathfrak{m}^{2}\right)^{*}, \mathfrak{m} / \mathfrak{m}^{2}\right)$, where $\mathfrak{m}$ is the kernel of the counit $\epsilon$ of $A(\mathcal{C})$.

Proof. Note that the set of all finite dimensional left $U(\mathfrak{g})$-modules is closed under finite direct sums and finite tensor products. Since every element of the restricted dual $U(\mathfrak{g})^{\circ}$ is represented by a coordinate function $c_{f, v}^{M}$ for some finite dimensional left $U(\mathfrak{g})$-module $M$, we have immediately that $A(\mathcal{C})$ is the restricted dual $U(\mathfrak{g})^{\circ}$. Moreover $\left(\left(\mathfrak{m} / \mathfrak{m}^{2}\right)^{*}, \mathfrak{m} / \mathfrak{m}^{2}\right)$ is a Lie bialgebra by 2.2 and 1.5 , and $\mathfrak{g}=\left(\mathfrak{m} / \mathfrak{m}^{2}\right)^{*}$ by $[7,7.11]$. Thus $\mathfrak{g}^{*}$ is equal to $\mathfrak{m} / \mathfrak{m}^{2}$ as a Lie algebra by (3) and (10). It follows that the Lie bialgebra $\left(\left(\mathfrak{m} / \mathfrak{m}^{2}\right)^{*}, \mathfrak{m} / \mathfrak{m}^{2}\right)$ is equal to $(\mathfrak{g}, \psi)=\left(\mathfrak{g}, \mathfrak{g}^{*}\right)$. 
Example 2.4. In the symplectic Lie algebra $\mathfrak{s p}_{4}$, set

$$
\begin{aligned}
& h_{1}=E_{11}-E_{22}-E_{33}+E_{44}, h_{2}=E_{22}-E_{44} \text {, } \\
& e_{1}=E_{12}-E_{43}, \quad e_{2}=E_{24}, \quad e_{3}=E_{14}+E_{23}, \quad e_{4}=E_{13} \text {, } \\
& f_{1}=E_{21}-E_{34}, \quad f_{2}=E_{42}, \quad f_{3}=E_{41}+E_{32}, \quad f_{4}=E_{31}
\end{aligned}
$$

(see $[5,8.3]$ for $\mathfrak{s p}_{4}$ ). Let $H$ be the subspace of $\mathfrak{s p}_{4}$ spanned by $h_{1}, h_{2}$ and let $\alpha_{1}, \alpha_{2} \in H^{*}$ be defined by

$$
\begin{array}{ll}
\alpha_{1}\left(h_{1}\right)=2, & \alpha_{2}\left(h_{1}\right)=-2 \\
\alpha_{1}\left(h_{2}\right)=-1, & \alpha_{2}\left(h_{2}\right)=2
\end{array}
$$

Then $e_{1}, e_{2}, e_{3}, e_{4}, f_{1}, f_{2}, f_{3}, f_{4}$ are weight vectors with weights

$$
\begin{aligned}
& \operatorname{wt}\left(e_{1}\right)=\alpha_{1}, \quad \operatorname{wt}\left(e_{2}\right)=\alpha_{2}, \quad \operatorname{wt}\left(e_{3}\right)=\alpha_{1}+\alpha_{2}, \quad \operatorname{wt}\left(e_{4}\right)=2 \alpha_{1}+\alpha_{2}, \\
& \operatorname{wt}\left(f_{1}\right)=-\alpha_{1}, \operatorname{wt}\left(f_{2}\right)=-\alpha_{2}, \quad \operatorname{wt}\left(f_{3}\right)=-\left(\alpha_{1}+\alpha_{2}\right), \quad \operatorname{wt}\left(f_{4}\right)=-\left(2 \alpha_{1}+\alpha_{2}\right) .
\end{aligned}
$$

Hence $\alpha_{1}, \alpha_{2}$ are positive simple roots. It is well-known that

$$
r=e_{1} \wedge f_{1}+2 e_{2} \wedge f_{2}+e_{3} \wedge f_{3}+2 e_{4} \wedge f_{4} \in \mathfrak{s p}_{4} \wedge \mathfrak{s p}_{4}
$$

satisfies the modified classical Yang-Baxter equation and gives the standard Lie bialgebra structure $\psi$ in $\mathfrak{s p}_{4}$ such that

$$
\begin{array}{ll}
\psi\left(h_{1}\right)=0, & \psi\left(h_{2}\right)=0, \\
\psi\left(e_{1}\right)=e_{1} \wedge h_{1}, & \psi\left(e_{2}\right)=2 e_{2} \wedge h_{2}, \\
\psi\left(e_{3}\right)=e_{3} \wedge h_{1}+2 e_{3} \wedge h_{2}-4 e_{1} \wedge e_{2}, & \psi\left(e_{4}\right)=2 e_{4} \wedge h_{1}+2 e_{4} \wedge h_{2}-2 e_{1} \wedge e_{3}, \\
\psi\left(f_{1}\right)=f_{1} \wedge h_{1}, & \psi\left(f_{2}\right)=2 f_{2} \wedge h_{2}, \\
\psi\left(f_{3}\right)=f_{3} \wedge h_{1}+2 f_{3} \wedge h_{2}-4 f_{1} \wedge f_{2}, & \psi\left(f_{4}\right)=2 f_{4} \wedge h_{1}+2 f_{4} \wedge h_{2}-2 f_{1} \wedge f_{3}
\end{array}
$$

(see [9, Exercise 4.1.11]).

The weight lattice $\mathbf{P}$ in $\mathfrak{s p}_{4}$ is a free abelian group with basis consisting of the fundamental dominant integral weights $\lambda_{1}, \lambda_{2}$, where $\lambda_{i}\left(h_{j}\right)=\delta_{i j}$ for $i, j=1,2$. Hence

$$
\alpha_{1}=2 \lambda_{1}-\lambda_{2}, \quad \alpha_{2}=-2 \lambda_{1}+2 \lambda_{2}
$$

The natural $\mathfrak{s p}_{4}$-module $V=\mathbf{k}^{4}$ is an irreducible highest weight module with highest weight $\lambda_{1}$. In fact, set

$$
v_{1}=\left(\begin{array}{l}
1 \\
0 \\
0 \\
0
\end{array}\right), v_{2}=\left(\begin{array}{l}
0 \\
1 \\
0 \\
0
\end{array}\right), v_{3}=\left(\begin{array}{l}
0 \\
0 \\
0 \\
1
\end{array}\right), v_{4}=\left(\begin{array}{c}
0 \\
0 \\
-1 \\
0
\end{array}\right) \in V .
$$

Then $v_{1}$ is a highest weight vector with highest weight $\lambda_{1}$ and

$$
\begin{aligned}
v_{1} \in V_{\lambda_{1}}, v_{2}=f_{1} v_{1} \in & V_{-\lambda_{1}+\lambda_{2}}, v_{3}=f_{2} v_{2} \in V_{\lambda_{1}-\lambda_{2}}, v_{4}=f_{1} v_{3} \in V_{-\lambda_{1}} . \\
& \left(v_{1} \stackrel{f_{1}}{\longrightarrow} v_{2} \stackrel{f_{2}}{\longrightarrow} v_{3} \stackrel{f_{1}}{\longrightarrow} v_{4}\right)
\end{aligned}
$$

Here we simply write $c_{f, v}$ for $c_{f, v}^{V}, v \in V, f \in V^{*}$. Observe that 
$v_{1}^{*}=\left(V^{*}\right)_{-\lambda_{1}}, v_{2}^{*}=v_{1}^{*} e_{1} \in\left(V^{*}\right)_{\lambda_{1}-\lambda_{2}}, v_{3}^{*}=v_{2}^{*} e_{2} \in\left(V^{*}\right)_{-\lambda_{1}+\lambda_{2}}, v_{4}^{*}=v_{3}^{*} e_{1} \in\left(V^{*}\right)_{\lambda_{1}}$

$$
\left(v_{1}^{*} \stackrel{e_{1}}{\longrightarrow} v_{2}^{*} \stackrel{e_{2}}{\longrightarrow} v_{3}^{*} \stackrel{e_{1}}{\longrightarrow} v_{4}^{*}\right)
$$

and

$$
\mathcal{C}=\left\{\mathbf{k}, V^{n}, V^{\otimes n} \mid n=1,2, \ldots\right\}
$$

is a class of $U\left(\mathfrak{s p}_{4}\right)$-modules closed under finite direct sums and finite tensor products. Thus $A(\mathcal{C})$ is a sub-Poisson bialgebra of the Poisson bialgebra $U\left(\mathfrak{s p}_{4}\right)^{\circ}$ by 2.2 . Set

$$
\begin{array}{rlrl}
h_{1}^{*} & =\overline{c_{v_{1}^{*}, v_{1}}-1}, & h_{2}^{*}=\overline{c_{v_{2}^{*}, v_{2}}+c_{v_{1}^{*}, v_{1}}-2}, & \\
x_{1}^{*}=\overline{c_{v_{1}^{*}, v_{2}}}, & x_{2}^{*}=\overline{c_{v_{1}^{*}, v_{3}}}, & y_{1}^{*}=\overline{c_{v_{2}^{*}, v_{1}}}, \quad y_{2}^{*}=\overline{c_{v_{3}^{*}, v_{1}}}
\end{array}
$$

in $\mathfrak{m} / \mathfrak{m}^{2}$, where $\mathfrak{m}=\operatorname{ker} \epsilon$. Let $S$ be the antipode of $U\left(\mathfrak{s p}_{4}\right)^{\circ}$. Since $V^{*} \cong V$ as a $U\left(\mathfrak{s p}_{4}\right)$-module and $\left.m \circ\left(\operatorname{id}_{U(\mathfrak{s p}}\right)^{*} \otimes S\right) \circ \Delta=\epsilon 1$, we have

$$
\begin{array}{lll}
\overline{c_{v_{3}^{*}, v_{4}}}=-\overline{c_{v_{1}^{*}, v_{2}}}, \quad \overline{c_{v_{2}^{*}, v_{4}}}=-\overline{c_{v_{1}^{*}, v_{3}}}, \quad \overline{c_{v_{2}^{*}, v_{3}}}=0, \quad \overline{c_{v_{1}^{*}, v_{4}}}=0, \\
\overline{c_{v_{4}^{*}, v_{3}}}=-\overline{c_{v_{2}^{*}, v_{1}}}, \quad \overline{c_{v_{3}^{*}, v_{1}}}=-\overline{c_{v_{4}^{*}, v_{2}}}, \quad \overline{c_{v_{3}^{*}, v_{2}}}=0, \quad \overline{c_{v_{4}^{*}, v_{1}}}=0 .
\end{array}
$$

It follows that $\mathfrak{m} / \mathfrak{m}^{2}$ is a 6 -dimensional Lie algebra with structure

$$
\begin{array}{lll}
{\left[h_{1}^{*}, h_{2}^{*}\right]=0,} & {\left[h_{1}^{*}, x_{1}^{*}\right]=-x_{1}^{*},} & {\left[h_{1}^{*}, x_{2}^{*}\right]=-x_{2}^{*},} \\
{\left[h_{1}^{*}, y_{1}^{*}\right]=-y_{1}^{*},} & {\left[h_{1}^{*}, y_{2}^{*}=-y_{2}^{*},\right.} & {\left[h_{2}^{*}, x_{1}^{*}\right]=0,} \\
{\left[h_{2}^{*}, x_{2}^{*}\right]=-2 x_{2}^{*},} & {\left[h_{2}^{*}, y_{1}^{*}=0,\right.} & {\left[h_{2}^{*}, y_{2}^{*}\right]=-2 y_{2}^{*},} \\
{\left[x_{1}^{*}, x_{2}^{*}\right]=0,} & {\left[y_{1}^{*}, y_{2}^{*}\right]=0,} & {\left[x_{i}^{*}, y_{j}^{*}\right]=0 \quad(i, j=1,2),}
\end{array}
$$

where $\left[h_{1}^{*}, h_{2}^{*}\right]=\overline{\left\{c_{v_{1}^{*}, v_{1}}-1, c_{v_{2}^{*}, v_{2}}+c_{v_{1}^{*}, v_{1}}-2\right\}} \in \mathfrak{m} / \mathfrak{m}^{2}$, etc. and the dual Lie algebra $\left(\mathfrak{m} / \mathfrak{m}^{2}\right)^{*}$ is a six dimensional Lie algebra with the following structure

$$
\begin{array}{llll}
{\left[h_{1}, h_{2}\right]=0,} & {\left[h_{1}, x_{1}\right]=2 x_{1},} & {\left[h_{1}, x_{2}\right]=0,} \\
{\left[h_{1}, y_{1}\right]=-2 y_{1},} & {\left[h_{1}, y_{2}\right]=0,} & & {\left[h_{2}, x_{1}\right]=-x_{1},} \\
{\left[h_{2}, x_{2}\right]=x_{2},} & {\left[h_{2}, y_{1}\right]=y_{1},} & & {\left[h_{2}, y_{2}\right]=-y_{2},} \\
{\left[x_{1}, x_{2}\right]=0,} & {\left[y_{1}, y_{2}\right]=0,} & & {\left[x_{1}, y_{1}\right]=h_{1},} \\
{\left[x_{1}, y_{2}\right]=0,} & {\left[x_{2}, y_{1}\right]=0,} & & {\left[x_{2}, y_{2}\right]=h_{1}+2 h_{2} .}
\end{array}
$$

Hence the Lie algebra $\left(\mathfrak{m} / \mathfrak{m}^{2}\right)^{*}$ is a Lie bialgebra with cobracket $\psi$ satisfying

$$
\begin{array}{ll}
\psi\left(h_{1}\right)=0, & \psi\left(h_{2}\right)=0, \\
\psi\left(x_{1}\right)=x_{1} \wedge h_{1}, & \psi\left(x_{2}\right)=x_{2} \wedge h_{1}+2 x_{2} \wedge h_{2}, \\
\psi\left(y_{1}\right)=y_{1} \wedge h_{1}, & \psi\left(y_{2}\right)=y_{2} \wedge h_{1}+2 y_{2} \wedge h_{2} .
\end{array}
$$

\section{References}

[1] K. A. Brown and K. R. Goodearl, Lectures on Algebraic Quantum Groups, Advanced Courses in Mathematics. CRM Barcelona. Birkhauser Verlag, Basel, 2002.

[2] V. Chari and A. Pressley, A Guide to Quantum Groups, Cambridge University Press, Cambridge, 1994.

[3] T. J. Hodges and T. Levasseur, Primitive ideals of $C_{q}[\mathrm{SL}(3)]$, Comm. Math. Phys. 156 (1993), no. 3, 581-605.

[4] T. J. Hodges, T. Levasseur, and M. Toro, Algebraic structure of multiparameter quantum groups, Adv. Math. 126 (1997), no. 1, 52-92. 
[5] J. Hong and S.-J. Kang, Introduction to Quantum Groups and Crystal Bases, Graduate Studies in Mathematics, 42. American Mathematical Society, Providence, RI, 2002.

[6] J. E. Humphreys, Linear Algebraic Groups, Graduate Texts in Mathematics, vol. 21, Springer-Verlag Inc., thirding printing, 1987.

[7] J. C. Jantzen, Lectures on Quantum Groups, Graduate Studies in Mathematics, vol. 6, American Mathematical Society, Providence, 1996.

[8] A. Joseph, Quantum Groups and Their Primitive Ideals, A series of modern surveys in mathematics, vol. 3. Folge·Band 29, Springer-Verlag, Berlin, 1995.

[9] L. I. Korogodski and Y. S. Soibelman, Algebras of Functions on Quantum Groups. Part I., Mathematical Surveys and Monographs, 56. American Mathematical Society, Providence, RI, 1998.

[10] L. A. Lambe and D. E. Radford, Introduction to the Quantum Yang-Baxter Equation and Quantum Groups: an algebraic approach, Mathematics and its Applications, 423. Kluwer Academic Publishers, Dordrecht, 1997.

SEI-Qwon OH

Department of Mathematics

Chungnam National University

Daejeon 305-764, Korea

E-mail address: sqoh@cnu.ac.kr

Eun-Hee Cho

Department of Mathematics

Chungnam National University

DAEJEON 305-764, KoreA

E-mail address: ehcho@cnu.ac.kr 